\title{
Bounds on exceptional Dehn filling II
}

\author{
IAN AGOL
}

\begin{abstract}
We show that there are at most finitely many one cusped orientable hyperbolic 3manifolds which have more than eight nonhyperbolic Dehn fillings. Moreover, we show that determining these finitely many manifolds is decidable.
\end{abstract}

57M50; 30F40

\section{Introduction}

Thurston demonstrated that if one has a hyperbolic knot complement, all but finitely many Dehn fillings give hyperbolic manifolds [46]. The example with the largest known number of nonhyperbolic Dehn fillings is the figure-eight knot complement, which has 10 fillings which are not hyperbolic. Call a Dehn filling exceptional if it is not hyperbolic. Previous authors have distinguished between hyperbolike manifolds, which are atoroidal and aspherical, and hyperbolic manifolds, defining an exceptional filling to be one which is not hyperbolike. But by the geometrization theorem [39; $40 ; 28 ; 35 ; 16]$, a manifold is hyperbolic if and only if it is hyperbolike, so we need no longer make this distinction. Bleiler and Hodgson [7] showed that there are at most 24 exceptional Dehn fillings, using Gromov and Thurston's $2 \pi$-theorem and estimates on cusp size due to Colin Adams [1]. We made an improvement on the $2 \pi$-theorem [3], independently discovered by Lackenby [29], and used improved lower bounds on cusp size due to Cao and Meyerhoff [15], to get an upper bound of 12 exceptional Dehn fillings. In this paper, we show that there are at most finitely many one cusped hyperbolic manifolds which have exceptional Dehn fillings $r_{1}, r_{2}$ such that $\Delta\left(r_{1}, r_{2}\right)>5$ (where $\Delta\left(r_{1}, r_{2}\right)$ denotes the intersection numbers of the slopes of the Dehn fillings). This theorem is sharp, in that the $(-2,3,8)$ pretzel link complement $W^{\prime}$ admits two exceptional Dehn fillings $r_{1}, r_{2}$ with $\Delta\left(r_{1}, r_{2}\right)=5$. By hyperbolic Dehn filling the other cusp of $W^{\prime}$, we see that there are infinitely many 3-manifolds with $\Delta\left(r_{1}, r_{2}\right)=5$. This theorem implies that there are only finitely many one cusped hyperbolic 3-manifolds with more than 8 exceptional Dehn fillings, since there can be at most 8 curves on a torus with $\Delta\left(r_{1}, r_{2}\right) \leq 5$. We also prove the existence of an algorithm which will determine the manifolds which have $\Delta\left(r_{1}, r_{2}\right)>5$ for exceptional Dehn fillings $r_{1}, r_{2}$, and therefore which manifolds may have more than 8 exceptional 
Dehn fillings. It is conjectured that there are only four orientable hyperbolic manifolds with two exceptional Dehn fillings $r_{1}, r_{2}$ so that $\Delta\left(r_{1}, r_{2}\right)>5$. These manifolds are the Dehn fillings $W(-1), W(5), W(5 / 2)$ and $W(-2)$ on one component of the Whitehead link complement $W$, and are conjectured by Gordon to be the only such examples [23, Conjecture 3.4]. Of these four examples, only the figure eight knot complement $W(-1)$ has more than 8 exceptional Dehn fillings. It is conjectured that there are only finitely many one cusped hyperbolic 3-manifolds with more than 6 exceptional Dehn fillings; see Kirby [27, Problem 1.77].

Remark In the process of preparation of this manuscript, Lackenby and Meyerhoff gave a proof that a one cusped hyperbolic 3-manifold has at most 10 exceptional Dehn fillings [30]. They also prove that the distance between exceptional fillings is at most 8 . Their approach uses extensive computation based on Gabai, Meyerhoff and Milley [20], as well as some improved estimates on lengths of slopes of exceptional Dehn fillings under certain geometric hypotheses. Our results imply that it ought to be possible to continue the computational approach even further.

Our theorem depends on results of Anderson, Canary, Culler and Shalen [5] which have been subsequently strengthened by a culmination of various results in Kleinian groups. In particular, we use the result that Kleinian groups are limits of geometrically finite Kleinian groups (proved independently by Ohshika [37] and Namazi and Souto [36]), which depends on the classification of Kleinian groups, including the tameness conjecture proved by the author [2] and Calegari and Gabai [14] (see also Soma [44] and Bowditch [10]) and the ending lamination conjecture proved by Brock, Canary and Minsky [12; 34], and generalizes many previous results on density of geometrically finite Kleinian groups in the space of Kleinian groups by Brock and Bromberg [13; 11], going back to work of Jørgensen on the space of punctured torus groups [26] and Thurston's double limit theorem [45; 38]. We remark that these arguments depend on the ending lamination conjecture for general Kleinian groups, which has been claimed by Brock, Canary and Minsky and is treated in the preprint of Bowditch [9] based on their approach. The key geometric consequence that we make use of is the following result stated in Theorem 3.4: if a maximal horocusp in an orientable hyperbolic manifold has volume $<\pi-\epsilon$ for $\epsilon>0$, then the volume of the manifold is uniformly bounded as a function of $\epsilon$.

Acknowledgements We thank the referee for many helpful suggestions, and Yi Liu for providing Figure 3.

This work was partially supported by NSF grant DMS-0504975. 


\section{Background and definitions}

Let $N$ be a hyperbolic 3 -manifold. Then $N \cong \mathbb{H}^{3} / \Gamma$ where $\Gamma \cong \pi_{1}(N)$ is a discrete torsion-free subgroup of $\operatorname{Isom}\left(\mathbb{H}^{3}\right)$, with $p: \mathbb{H}^{3} \rightarrow N$ the covering map. We will assume that the reader is familiar with Margulis' constant $\epsilon$ and the thickthin decomposition in the case of hyperbolic 3-manifolds; see Thurston's notes [46, Chapter 5] for an introduction to this. The following definitions are needed only for part of the proof of Lemma 3.2. If $g$ is a loxodromic isometry of hyperbolic 3-space $\mathbb{H}^{3}$, we shall let $A_{g}$ denote the hyperbolic geodesic which is the axis of $g$. The cylinder about $A_{g}$ of radius $r$ is the open set $Z_{r}(g)=\left\{x \in \mathbb{H}^{3} \mid \operatorname{dist}\left(x, A_{g}\right)<r\right\}$. If $C$ is a simple closed geodesic in $N$ then there is a primitive loxodromic isometry $g \in \Gamma$ with $p\left(A_{g} /\langle g\rangle\right)=C$. For any $r>0$, the projection $p\left(Z_{r}(g) /\langle g\rangle\right)$ of $Z_{r}(g)$ under the covering projection is a neighborhood of $C$ in $N$. By the Margulis lemma, for sufficiently small $r>0$ we have

$$
\left\{h \in \Gamma \mid h\left(Z_{r}(g)\right) \cap Z_{r}(g) \neq \varnothing\right\}=\langle g\rangle .
$$

Let $R$ denote the supremum of the set of $r$ for which this condition holds ( $R$ depends on $C$ ). We define tube $(C)=Z_{R}(g) /\langle g\rangle$ to be the maximal tube about $C$. A set $U$ is said to be precisely invariant under the group $\Gamma$ if for every $g \in \Gamma$, either $g(U)=U$ or $g(U) \cap U=\varnothing$, which is equivalent to saying that $U / \operatorname{stab}(U)$ embeds in $\mathbb{H}^{3} / \Gamma$. Then tube $(C)$ is the largest precisely invariant tube about $C$.

There is a similar situation when we have a maximal parabolic subgroup $P<\Gamma$. We define an open horoball to be a subset of $\mathbb{H}^{3}$ isometric to $\{(z, t) \mid z \in \mathbb{C}, t>1\}$, where $\mathbb{H}^{3}=\{(z, t) \mid t>0\} \subset \mathbb{C} \times \mathbb{R}$ is the upper half-space model of the hyperbolic 3-space. By the Margulis lemma there is an open horoball $\tilde{\mathcal{H}} \subset \mathbb{H}^{3}$ so that $P=$ $\{g \in \Gamma \mid g(\tilde{\mathcal{H}})=\tilde{\mathcal{H}}\}$. Choose $\tilde{\mathcal{H}}$ maximal subject to the condition that $\{h \in \Gamma \mid$ $h(\tilde{\mathcal{H}}) \cap \widetilde{\mathcal{H}} \neq \varnothing\}=P$ (that is, $\widetilde{\mathcal{H}}$ is a maximal precisely invariant horocusp for $\Gamma$ such that $\operatorname{stab}(\widetilde{\mathcal{H}})=P$ ). Then we call $p(\tilde{\mathcal{H}})=\mathcal{H} \cong \tilde{\mathcal{H}} / P$ a maximal horocusp. If $P \cong \mathbb{Z}^{2}$, then $\mathcal{H} \cong S^{1} \times S^{1} \times \mathbb{R}$, and if $P \cong \mathbb{Z}$, then $\mathcal{H} \cong S^{1} \times \mathbb{R}^{2}$. Since $\mathcal{H}$ is maximal subject to this condition, there exists $\gamma \in \Gamma-P$ so that $\gamma(\partial \tilde{\mathcal{H}}) \cap \partial \tilde{\mathcal{H}}=x$. We may assume that $\Gamma$ is normalized by a conjugation so that $P$ fixes $\infty$, and $\partial \tilde{\mathcal{H}}$ is a plane of height one, so that $\gamma(\tilde{\mathcal{H}})$ is a ball of height 1 . Note that $\gamma^{-1}(\partial \widetilde{\mathcal{H}}) \cap \partial \tilde{\mathcal{H}}=\gamma^{-1}(x)$ will also be a point of tangency. Then $\gamma^{-1}(\tilde{\mathcal{H}})$ is also a horoball of height one call the Adams horoball [1] (see Figure 1). We will call the group $B=\langle P, \gamma\rangle$ a bicuspid group (see Agol, Culler and Shalen [4, Lemma 6.2]), so called because it corresponds to a point in $\mathbb{H}^{3}$ where the closure of two maximal horoballs meet, corresponding to two preimages of a neighborhood of a cusp of $N$. In this paper, when $\mathbb{Z}^{2} \cong P$, we will use the notation $P=\langle\alpha, \beta\rangle$, where $\alpha, \beta$ are parabolic elements. Then $B$ is free bicuspid 


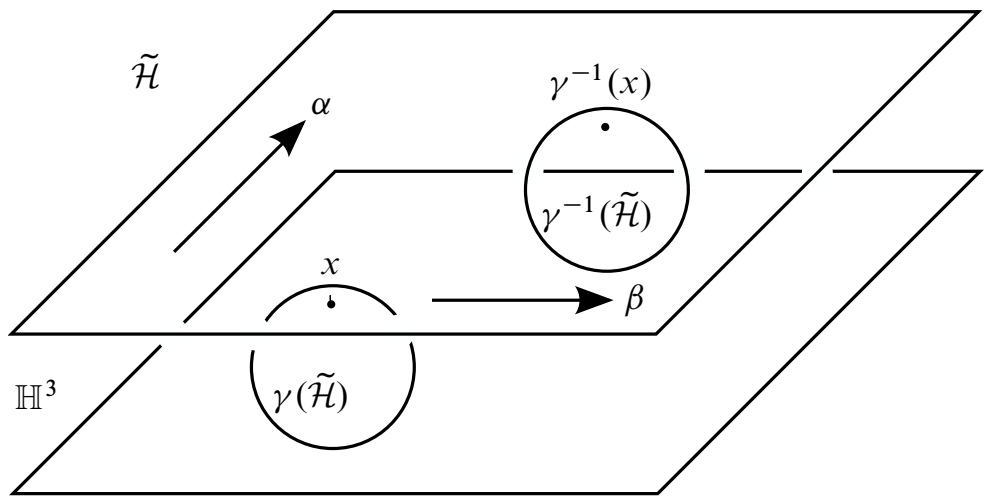

Figure 1: A bicuspid group associated to a maximal horocusp

if $B \cong P *\langle\gamma\rangle \cong \mathbb{Z}^{2} * \mathbb{Z}$. For any cusp of $N$ with associated maximal horocusp $\mathcal{H}$, there will be finitely many bicuspid subgroups of $\Gamma$ up to conjugacy in one-to-one correspondence with the $\Gamma$ orbits of points $x \in \mathbb{H}^{3}$ so that $x=\gamma(\partial \tilde{\mathcal{H}}) \cap \partial \tilde{\mathcal{H}}$ for some $\gamma \in \Gamma-P$. One may think of each of these points as "self-tangencies" of $\partial \mathcal{H}$.

Here we develop a normalization for the generators of the bicuspid group $B$ which will be used in examples and in the proof of Theorem 5.5. Let $N=\mathbb{H}^{3} / B$. We have $A=\operatorname{Area}(\partial \mathcal{H})=2 \operatorname{Vol}(\mathcal{H})$. As mentioned in the previous paragraph, we assume that $\tilde{\mathcal{H}}$ is normalized to be centered at $\infty$ and to have height 1 in the upper half-space model. The generators $\alpha, \beta$ of $P$ translate by complex numbers $a, b \in \mathbb{C}$ in $\partial \tilde{\mathcal{H}}=\mathbb{C}$, which has the standard Euclidean metric induced from the hyperbolic metric of $\mathbb{H}^{3}$. We may assume the generators for the parabolic subgroup $P$ are chosen so that $\alpha$ represents the shortest closed path in $\partial \mathcal{H}$, and $\beta$ the next shortest path, by choosing $b / a$ to lie in the standard fundamental domain for the moduli space of tori. Also, since $\alpha \gamma(\tilde{\mathcal{H}}) \cap \gamma(\tilde{\mathcal{H}})=\varnothing$, we must have $|a| \geq 1$ (this is an observation of Adams [1]; see Figure 1). Moreover, by the normalization, $|b| \leq 2 A / \sqrt{3}$ as follows from an analysis of the fundamental domain of moduli space. Conjugating $B$ by a parabolic or elliptic element fixing $\infty$, and taking $\gamma^{-1}(\infty)$ to 0 , we may normalize $\alpha, \beta, \gamma$ as elements in $\mathrm{SL}_{2} \mathbb{C}$ such that $\tilde{\mathcal{H}}$ is the horoball centered at $\infty$ of height $1, \gamma(0)=\infty$, and

$$
\alpha=\left(\begin{array}{ll}
1 & a \\
0 & 1
\end{array}\right), \quad \beta=\left(\begin{array}{ll}
1 & b \\
0 & 1
\end{array}\right), \quad \gamma=\left(\begin{array}{cc}
c & -1 \\
1 & 0
\end{array}\right),
$$

where $1 \leq|a| \leq|b| \leq 2 A / \sqrt{3}$. Further multiplication of $\gamma$ on the left by elements of $P$ allows us to assume that $c$ lies in a fundamental domain for the action of $P$ on $\mathbb{C}$ consisting of points closest to 0 , and therefore that $|c| \leq|b|$ (this requires a bit of analysis of fundamental domains of tori, but is not hard to show, and the precise 
estimate is not important for our argument). We will denote the parameter space by

$$
\mathcal{P}_{A}=\{(a, b, c)|1 \leq| a|\leq| b|\leq 2 A / \sqrt{3},| c|\leq| b \mid\} .
$$

\section{Maximal embedded cusps of hyperbolic manifolds}

The following result generalizes [25, Theorem IV.4.1] to Kleinian groups with cusps. This lemma is used in the proof of Lemma 5.3. One could give a purely topological proof, but we choose to make use of some Kleinian group theory.

Lemma 3.1 If $Q$ is a bicuspid group of infinite covolume, then $Q$ is free bicuspid.

Proof Since $\mathbb{H}^{3} / Q$ has infinite volume and is finitely generated, there is a compact 3-manifold with boundary $N$ such that $\mathbb{H}^{3} / Q \cong \operatorname{int}(N)$ (by tameness [2; 14]). Also, $\beta_{1}(N)=\beta_{1}(Q) \leq 3$. Since bicuspid groups are nonelementary, $\chi(N)<0$. Thus $N$ has a boundary component $T$ which is a torus, and a boundary component $\Sigma$ with $\chi(\Sigma)<0$. By half-lives, half-dies, $\Sigma$ must be a genus 2 surface, since $\beta_{1}(T)+$ $\beta_{1}(\Sigma) \leq \beta_{1}(\partial N) \leq 6$. The space of characters $X$ of discrete faithful representations of $\pi_{1}(N) \rightarrow \operatorname{PSL}(2, \mathbb{C})$ has complex dimension 3 (see Bers [6] and Marden [32, Theorem 10.8]). We also see that this is the dimension of the variety of characters of $\mathbb{Z}^{2} * \mathbb{Z}$ which are rank 2 parabolic on $\mathbb{Z}^{2}$. But the character variety of $N$ must be a subvariety of the same dimension, which means that $\pi_{1}(N)$ must have no relations among the bicuspid generators, and is therefore free.

The following geometric result is made possible by recent advances in Kleinian groups, and generalizes [4, Lemma 4.3].

Lemma 3.2 Let $\langle\alpha, \beta, \gamma\rangle$ be a free bicuspid group, with maximal horocusp $\mathcal{H}$. Then $\operatorname{Vol}(\mathcal{H}) \geq \pi$.

Proof To prove the theorem, first we approximate $Q$ by geometrically finite groups $Q_{i}$ such that $Q_{i}=\left\langle\alpha_{i}, \beta_{i}, \gamma_{i}\right\rangle$ and such that $\alpha_{i} \rightarrow \alpha, \beta_{i} \rightarrow \beta, \gamma_{i} \rightarrow \gamma$. Denote by $N_{i}=\mathbb{H}^{3} / Q_{i}$ the hyperbolic manifold which is homeomorphic to the interior of a compression body which is the boundary connect sum $\left(T^{2} \times I\right) \amalg\left(S^{1} \times D^{2}\right) \cong \bar{N}_{i}$. Denote $S_{i}$ to be the torus boundary component of $\bar{N}_{i}$. In fact, one may assume that in the groups $Q_{i}$, the only parabolic elements are conjugate into $\left\langle\alpha_{i}, \beta_{i}\right\rangle$. This is possible by the fact that geometrically finite groups without rank one parabolics are dense in the space of all Kleinian groups in the algebraic topology [37, Theorem 1.1; 36, Theorem 1.1]. We may assume that $N_{i}$ contains a maximal embedded horocusp neighborhood $\mathcal{H}_{i}$ such that $\operatorname{Vol}\left(\mathcal{H}_{i}\right) \rightarrow \operatorname{Vol}(\mathcal{H})$. 
Next, we proceed as in Lemma 4.3 of [4]. Suppose that $\left(P_{j}\right)$ is an infinite sequence of distinct hyperbolic manifolds obtained by Dehn filling $N_{i}$ along $S_{i}$ using Thurston's hyperbolic Dehn surgery theorem for geometrically finite manifolds (see Bonahon and Otal [8] or Comar [17]). Then $\pi_{1}\left(P_{j}\right)$ is free two generator, and the manifolds $\left(P_{j}\right)$ converge geometrically to $N_{i}$. Moreover, the core curve of the Dehn filling $P_{j}$ of $N_{i}$ is isotopic to a geodesic $C_{j}$ in $P_{j}$. The length $L_{j}$ of $C_{j}$ tends to 0 as $j \rightarrow \infty$; and the sequence of maximal tubes (tube $\left(C_{j}\right)$ ), $j \geq 1$ converges geometrically to $\mathcal{H}_{i}$. In particular $\lim _{j \rightarrow \infty} \operatorname{Vol}\left(\operatorname{tube}\left(C_{j}\right)\right)=\operatorname{Vol}\left(\mathcal{H}_{i}\right)$. According to [4, Corollary 4.2], since $\pi_{1}\left(P_{j}\right)$ is free, $\log 3$ is a strong Margulis number for each of the hyperbolic manifolds $P_{j}$. It therefore follows from [5, Corollary 10.5] that $\operatorname{Vol}\left(\operatorname{tube}\left(C_{j}\right)\right)>V\left(L_{j}\right)$, where $V$ is an explicitly defined function such that $\lim _{x \rightarrow 0} V(x)=\pi$. In particular, this shows that $\operatorname{Vol}\left(\mathcal{H}_{i}\right) \geq \lim _{j \rightarrow \infty} V\left(L_{j}\right) \geq \pi$. Now, since $\mathcal{H}_{i}$ converges geometrically to $\mathcal{H}$, we conclude that $\operatorname{Vol}(\mathcal{H}) \geq \pi$.

Remark The estimates of [5] depend on a paradoxical decomposition argument for a 2-generator free group acting on $\mathbb{H}^{3}$ given in [19]. We believe that it should be possible to give a more direct argument for the previous lemma by analyzing an appropriate generalization of the paradoxical decomposition for free bicuspid groups.

Example Consider the group $\Gamma=\langle\alpha, \beta, \gamma\rangle$, where

$$
\alpha=\left(\begin{array}{ll}
1 & 4 \\
0 & 1
\end{array}\right), \quad \beta=\left(\begin{array}{cc}
1 & 1+i \sqrt{3} \\
0 & 1
\end{array}\right), \quad \gamma=\left(\begin{array}{cc}
2 & -1 \\
1 & 0
\end{array}\right) .
$$

One may show that this group is free as follows. Consider the geodesic planes in the upper half space model of $\mathbb{H}^{3}$ bounding the circles $\{|z-c|=2 \mid c \in \mathbb{Z} 2+\mathbb{Z}(1+i \sqrt{3})\}$, and cut out the open half-spaces disjoint from $\infty$ bounded by these planes to obtain a region $R \subset \mathbb{H}^{3}$. These bounding circles have two orbits under the group $\langle\alpha, \beta\rangle$, with representatives at centers $c=0,2$, and thus $R /\langle\alpha, \beta\rangle$ will be homeomorphic to $T^{2} \times \mathbb{R}$ with two geodesic disks in its boundary. Under the map $\gamma$, the circle $|z|=2$ is sent to the circle $|z-2|=2$, and therefore $\gamma$ maps the corresponding planes bounding these circles to themselves. Thus, $N=\mathbb{H}^{3} / \Gamma$ is obtained from $R /\langle\alpha, \beta\rangle$ by gluing the two geodesic planes in the boundary using the identification given by $\gamma$. This has the effect of adding a handle onto $T^{2} \times \mathbb{R}$, and therefore $N$ is homeomorphic to the interior of a compression body with fundamental group identified with $\Gamma \cong\langle\alpha, \beta\rangle *\langle\gamma\rangle$. Thus, we see that $\Gamma$ is free bicuspid. If $\tilde{\mathcal{H}}$ is the horosphere centered at $\infty$ of height one in $\mathbb{H}^{3}$, then $\tilde{\mathcal{H}} /\langle\alpha, \beta\rangle$ embeds as a cusp $\mathcal{H} \subset N$, since it is disjoint from the geodesic planes given above. We have $\operatorname{Vol}(\mathcal{H})=2 \sqrt{3}=3.46 \ldots$ Thus, the bound given in Lemma 3.2 is fairly close to optimal.

Here is another way to find free bicuspid groups. 
Lemma 3.3 Let $M$ be an orientable hyperbolic 3-manifold with a maximal horocusp $\mathcal{H}$ and an associated bicuspid subgroup $B<\pi_{1}(M)$. If $\operatorname{rank} H_{1}(M ; \mathbb{Z} / p \mathbb{Z}) \geq 4$, where $p$ is prime and
(1) $M$ has one cusp and $p>3$ or
(2) $M$ has at least two cusps and $p \geq 2$,

then $B$ is free bicuspid, and therefore $\operatorname{Vol}(\mathcal{H}) \geq \pi$.

Proof This essentially follows from the arguments of [4, Lemma 4.3, Proposition 5.3, Proposition 6.3]. First, assume that $M$ has 4 or more cusps, or $\operatorname{rank} H_{1}(M ; \mathbb{Q}) \geq 4$. Then there exists a map $\phi: \pi_{1}(M) \rightarrow \mathbb{Z}^{4}$. As $\operatorname{rank} B /[B, B] \leq 3, \operatorname{rank} \phi\left(\pi_{1}(B)\right) \leq 3$, and therefore $B$ has infinite index in $\pi_{1}(M)$. Thus, $B$ is free bicuspid (see Lemma 3.1).

Now suppose that rank $H_{1}(M ; \mathbb{Z} / p \mathbb{Z}) \geq 4$, and we have a map $\phi: \pi_{1}(M) \rightarrow(\mathbb{Z} / p \mathbb{Z})^{4}$. Then $\operatorname{rank} \phi(B) \leq 3$, so there exists a map $\pi_{1}(M) \rightarrow(\mathbb{Z} / p \mathbb{Z})^{4} / \phi(B) \rightarrow \mathbb{Z} / p \mathbb{Z}$. Take the kernel of this map to get a $p$-fold cover $\widetilde{M} \rightarrow M$ such that $B \leq \pi_{1}(\widetilde{M})$. Under the hypotheses of the theorem, $\widetilde{M}$ has at least 4 cusps, and by the previous paragraph, $B$ must be free bicuspid. Then $\operatorname{Vol}(\mathcal{H}) \geq \pi$ by Lemma 3.2.

We did a search of manifolds in the SnapPea census [47], and found that the manifold $M=\mathrm{v} 1902$ has an embedded cusp of volume $3.238 \ldots$. Moreover, the bicuspid group associated to $\mathrm{v} 1902$ is free. One may see this by finding an irregular 3-fold cover $\widetilde{M} \rightarrow M$ (cover 8 in SnapPea's notation) which has 3 maximal cusps with the same volume as $M$, and has $H_{1}(\widetilde{M})=\mathbb{Z}^{3}+\mathbb{Z} / 3 \mathbb{Z}$ (see Figure 2). It follows that the bicuspid subgroup of $\pi_{1} M$ is free, since it lifts to $\pi_{1} \widetilde{M}$, and any bicuspid subgroup of $\pi_{1} \widetilde{M}$ is free by Lemma 3.3 since $\operatorname{rank} H_{1}(\widetilde{M} ; \mathbb{Z} / 3 \mathbb{Z})=4$.

In fact, there will be an optimal constant $\pi \leq R<3.238 \ldots$ such that the quotient manifold of every free bicuspid group will have an embedded horocusp $\mathcal{H}$ with $\operatorname{Vol}(\mathcal{H}) \geq R$. It is an interesting question to obtain better upper and lower bounds on $R$. The following theorem will be reproved in Section 5; however we include this proof here as it is a bit more direct than the argument of Theorem 5.5. The following theorem would hold with $R$ replacing $\pi$.

Theorem 3.4 For $\epsilon>0$, there exists a constant $V(\epsilon)$ such that if $N$ is an orientable hyperbolic 3-manifold with a maximal horocusp $\mathcal{H}$ with $\operatorname{Vol}(\mathcal{H})<\pi-\epsilon$, then $\operatorname{Vol}(N)<V(\epsilon)$. 


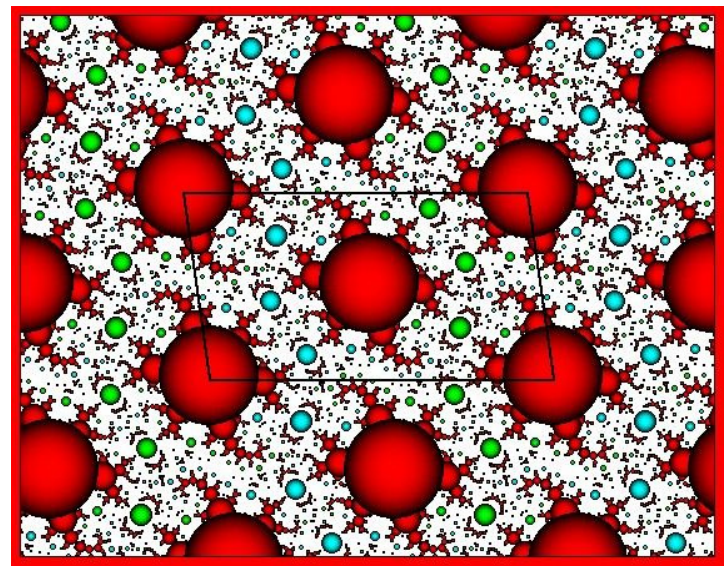

Figure 2: Pattern of horoballs in $\mathbb{H}^{3}$ for the manifold v1902 3-fold cover 8 from the SnapPea census with cusp volume $3.238 \ldots$

Proof Let $Q<\pi_{1} N$ be a bicuspid subgroup corresponding to the maximal horocusp $\mathcal{H}$. First, suppose that $Q$ is a free product $Q=\langle\alpha, \beta\rangle *\langle\gamma\rangle \cong(\mathbb{Z}+\mathbb{Z}) * \mathbb{Z}$. Then $\operatorname{Vol}(\mathcal{H}) \geq \pi$ by Lemma 3.2, contradicting the assumption that $\operatorname{Vol}(\mathcal{H})<\pi-\epsilon$.

Thus, we may assume that $Q$ is not a free product. Then $Q$ must be indecomposable, and in fact $\mathbb{H}^{3} / Q$ must be finite volume, by Lemma 3.1. We get a contradiction in this case by taking a geometric limit. Suppose we have $\Gamma_{j}<\operatorname{PSL}(2, \mathbb{C})$ with $N_{j}=\mathbb{H}^{3} / \Gamma_{j}$, such that $\operatorname{Vol}\left(N_{j}\right) \rightarrow \infty$ and maximal bicuspid subgroups $Q_{j}<\Gamma_{j}$, such that $Q_{j}$ is not free bicuspid and $\operatorname{Vol}\left(\mathcal{H}_{j}\right)<\pi-\epsilon$, where $\mathcal{H}_{j}$ is a maximal cusp of $\mathbb{H}^{3} / Q_{j}$. Then $\operatorname{Vol}\left(\mathbb{H}^{3} / Q_{j}\right)<\infty$ by Lemma 3.1 , so $\left[\Gamma_{j}: Q_{j}\right]<\infty$. Normalize $Q_{j}$ as a standard bicuspid group with $\mathcal{H}_{j} \subset \mathbb{H}^{3} / Q_{j}$ the maximal cusp, and with self-tangency point of $x_{j} \in \partial \mathcal{H}_{j} \subset \mathbb{H}^{3} / Q_{j}$ as in Equation (1).

$$
\alpha_{j}=\left(\begin{array}{cc}
1 & a_{j} \\
0 & 1
\end{array}\right), \quad \beta_{j}=\left(\begin{array}{cc}
1 & b_{j} \\
0 & 1
\end{array}\right), \quad \gamma_{j}=\left(\begin{array}{cc}
c_{j} & -1 \\
1 & 0
\end{array}\right),
$$

where we assume that the generators are normalized so that $\left(a_{j}, b_{j}, c_{j}\right) \in \mathcal{P}_{2 \pi}$.

Since by the Margulis lemma the injectivity radius $\operatorname{inj}\left(x_{j}\right)$ is bounded below, we may take a subsequence $j_{k}$ such that $\left(\mathbb{H}^{3} / Q_{j_{k}}, x_{j_{k}}\right)$ converges geometrically to $\left(M_{\infty}, x_{\infty}\right)$, where $\mathcal{H}_{\infty} \subset M_{\infty}$ is a maximal cusp with self-tangency point $x_{\infty}$ and $\operatorname{Vol}\left(M_{\infty}\right)=\infty$, and so that $\left(a_{j_{k}}, b_{j_{k}}, c_{j_{k}}\right)$ converges to a point $\left(a_{\infty}, b_{\infty}, c_{\infty}\right) \in \mathcal{P}_{2 \pi}$. We are using the standard fact here that the algebraic limit of a sequence of groups is a subgroup of the fundamental group of the geometric limit (or perhaps the limit in the Chabauty topology). Then $Q_{j}$ converges algebraically to a bicuspid subgroup $\left\langle\alpha_{\infty}, \beta_{\infty}, \gamma_{\infty}\right\rangle=Q_{\infty}<\pi_{1}\left(M_{\infty}\right)$ such that $\mathcal{H}_{\infty}$ lifts to a maximal cusp in $\mathbb{H}^{3} / Q_{\infty}$ 
and $\operatorname{Vol}\left(\mathbb{H}^{3} / Q_{\infty}\right)=\infty$. By Lemma $3.1, Q_{\infty} \cong(\mathbb{Z}+\mathbb{Z}) * \mathbb{Z}$, so that $Q_{\infty}$ is a free bicuspid group. The cusps $\mathcal{H}_{j} \rightarrow \mathcal{H}_{\infty}$ in the Gromov-Hausdorff topology. Since $\operatorname{Vol}\left(\mathcal{H}_{\infty}\right) \geq \pi$ by Lemma 3.2, we see that there is $k$ such that $\operatorname{Vol}\left(\mathcal{H}_{j_{k}}\right)>\pi-\epsilon$. This gives a contradiction, so we see that the assumption that $\operatorname{Vol}\left(\mathbb{H}^{3} / Q_{j}\right) \rightarrow \infty$ is false, and thus there exists $V(\epsilon)$ such that $\operatorname{Vol}\left(N_{j}\right)<V(\epsilon)$.

Remark A similar argument to the previous theorem shows that there is a constant $V$ such that if $M$ is a hyperbolic 3-manifold with Margulis constant $<\log 3$, then $\operatorname{Vol}(M)<V$ by applying [19]. It would be interesting to get some idea of the distribution of Margulis constants $<\log 3$.

\section{Exceptional Dehn fillings}

In this section, we prove the main theorem of the paper.

Theorem 4.1 There are only finitely many one cusped orientable hyperbolic 3-manifolds of finite volume $N$ such that $N$ has two exceptional Dehn fillings $a_{1}, a_{2}$ so that $\Delta\left(a_{1}, a_{2}\right)>5$.

Proof Fix some $\epsilon<\pi-3$. If $\operatorname{Vol}(N) \geq V(\epsilon)$, where $V(\epsilon)$ is the constant from Theorem 3.4, then $N$ has a maximal horocusp $\mathcal{H} \subset N$ so that $\operatorname{Vol}(\mathcal{H}) \geq \pi-\epsilon>3$. Then $\operatorname{Area}(\partial \overline{\mathcal{H}})>6$. It was proven by Agol [3, Theorem 6.2] and Lackenby [29, Theorem 3.1] that if $a$ is a slope such that $\bar{N}(a)$ is an exceptional Dehn filling, then $l(a) \leq 6$. Thus by the proof of [3, Theorem 8.1], if $a_{1}, a_{2}$ are two slopes on $\partial \bar{N}$ so that $\bar{N}\left(a_{i}\right)$ are exceptional Dehn fillings, then $\Delta\left(a_{1}, a_{2}\right) \leq 6^{2} / \operatorname{Area}(\partial \overline{\mathcal{H}})<6$. Thus, $N$ does not violate the theorem.

Suppose the theorem is false. Then there is an infinite sequence of orientable one cusped hyperbolic 3-manifolds $\left(N_{i}\right)_{i \in \mathbb{N}}$ such that $i \rightarrow \infty$ and $N_{i}$ has two exceptional Dehn fillings of distance $>5$. By the previous paragraph, we may assume that $\operatorname{Vol}\left(N_{i}\right)<V(\epsilon)$. Let $\mathcal{H}_{i}$ be a maximal cusp neighborhood in $N_{i}$. By [46, Theorem 5.12.1], we may choose a subsequence $J \subset \mathbb{N}$ and an orientable hyperbolic 3-manifold $M$ with $\geq 2$ rank two cusps so that each $N_{i}, i \in J$ is obtained by hyperbolic Dehn filling on $M$, and $N_{i}$ converges to $M$ in the Gromov-Hausdorff topology. Moreover, $M$ has a distinguished cusp with maximal cusp neighborhood $\mathcal{H}$ such that as $i \rightarrow \infty, i \in J, \mathcal{H}_{i} \rightarrow \mathcal{H}$ in the Gromov-Hausdorff topology. In fact, since $\operatorname{Vol}(\mathcal{H}) \leq \pi-\epsilon$, we may assume that $M$ has at most three cusps, by Lemma 3.3. This observation is not necessary for the proof, but makes the notation of the proof simpler. By [22, Theorem 1.3], the distance between exceptional filling slopes on the cusp $\mathcal{H}$ of $M$ is $\leq 5$. 
Let $\partial \bar{M}=T_{0} \cup T_{1} \cup T_{2}$, where $T_{0}$ corresponds to $\mathcal{H}$. For slopes $q_{j} \in \mathbb{Q} \cup \infty \cup *$, $j=0,1,2$, let $M\left(q_{0}, q_{1}, q_{2}\right)$ be Dehn filling on the boundary component $T_{i}$ with slope $q_{i}$, where the $*$ denotes that the boundary component is unfilled. Let $\overline{N_{i}}=$ $M\left(*, q_{i, 1}, q_{i, 2}\right), i \in J$. For each $0 \leq j \leq 2$, there is a finite subset $E_{j} \subset \mathbb{Q} \cup \infty$ so that if $q_{j} \notin E_{j}$, then $M\left(q_{0}, q_{1}, q_{2}\right)$ is hyperbolic [46, Theorem 5.8.2]. For $i \in J$ large enough, $q_{i, j} \notin E_{j}$, for $j=1,2$. This implies that the only nonhyperbolic Dehn fillings on $N_{i}$ must correspond to a subset of the slopes $E_{0}$. Let $E_{h} \subset E_{0}$ be such that $t \in E_{h}$ if and only if $t \in E_{0}$ and $M(t, *, *)$ is hyperbolic. For $t_{1}, t_{2} \in E_{0}-E_{h}$, we have $\Delta\left(t_{1}, t_{2}\right) \leq 5$ by [22, Theorem 1.3] as noted above. For each slope $t \in E_{h}$ there are finite subsets $E_{t, j} \subset \mathbb{Q} \cup \infty$ so that $M\left(t, q_{1}, q_{2}\right)$ is hyperbolic if $q_{j} \notin E_{t, j}$ (again by [46, Theorem 5.8.2]). Let $F_{j}=E_{j} \cup_{t \in E_{h}} E_{t, j}$. Then if $q_{i, j} \notin F_{j}$ and $t \in E_{h}$, then $N_{i}(t)$ is also hyperbolic. Therefore, we see that for $i$ large, the distance between two exceptional slopes of $N_{i}$ is at most 5. This gives a contradiction to our assumption that there is a sequence of manifolds contradicting the theorem with volumes bounded by $V(\epsilon)$, thus proving the theorem.

Corollary 4.2 There are only finitely many one cusped orientable hyperbolic 3manifolds of finite volume $N$ such that $N$ has $>8$ exceptional Dehn fillings.

Proof By [3, Lemma 8.2], if the distance between exceptional Dehn fillings is $\leq 5$, take the next largest prime and add one, to conclude there are at most 8 exceptional Dehn fillings on $N$.

\section{An algorithm to find exceptional Dehn fillings}

We would like to classify the finitely many manifolds with two exceptional Dehn fillings of distance $>5$ given by Theorem 4.1. Since the proof of Theorem 3.4 is by contradiction, it's not clear that there is a procedure one could run which would for a given $\epsilon$ identify all of the manifolds which have an embedded cusp of volume $<\pi-\epsilon$. Thus, we must devise a method to classify manifolds with a small volume cusp. This is similar to the procedures implemented in $[21 ; 20]$, which find manifolds with small radius tubes around a short geodesic, or small volume cusped manifolds. We modify their approach to show that finding the manifolds with exceptional fillings in Theorem 4.1 and Corollary 4.2 is decidable. First, we need some preliminary results.

Theorem 5.1 Given a finite volume hyperbolic 3-manifold $M$, there is an algorithm which will determine the set of all exceptional Dehn fillings on $M$ for which any proper sub-Dehn filling is hyperbolic. 
Proof We approximate the hyperbolic structure on the cusped manifold well enough to determine the area and modulus (for example using interval arithmetic ) of simultaneously embedded equal sized horocusp neighborhoods of all the cusps, up to some small error. This uses the algorithms to compute Ford domains in Riley [42], Hildebrand and Weeks [24] and SnapPea [48] (see also Manning [31]). We determine which slopes on each cusp have length $\leq 6$, and therefore could lead to nonhyperbolic Dehn fillings by $[3 ; 29]$. We perform all of the Dehn fillings along these short slopes, determining which ones result in hyperbolic manifolds using the algorithms described in [42;48;31], then repeat. At each stage we get hyperbolic manifolds with fewer cusps, and so this process eventually terminates with a finite collection of cusped hyperbolic manifolds along with a finite collection of slopes associated to each cusp, so that every exceptional Dehn filling on $M$ is obtained by Dehn filling on one of the slopes associated to one of the members of this finite collection.

Lemma 5.2 Let $N$ be a hyperbolic 3-manifold, and $R \subset N$ be a connected region, such that $\operatorname{im}\left\{\pi_{1}(R) \rightarrow \pi_{1}(N)\right\}$ is elementary. Then $\operatorname{Vol}(R) \leq \frac{1}{2} \operatorname{Area}(\partial R)$.

Proof This follows from the isoperimetric inequality for $\mathbb{H}^{3}$ plus the fact that the image of $\pi_{1} R$ in $\pi_{1} N$ is amenable. Alternatively, one may also use the method of [41, Lemma 3.2, Theorem 4.1], which is essentially a calibration argument.

Lemma 5.3 Suppose $Q=\langle\alpha, \beta, \gamma\rangle$ is a (discrete torsion-free) bicuspid group which is not free. Let $N=\mathbb{H}^{3} / Q$. Let $w(x, y, z) \in(\langle x\rangle \times\langle y\rangle) *\langle z\rangle$ be a cyclically reduced word, such that $w(\alpha, \beta, \gamma)=1 \in Q$. Let $d(w)$ be the number of occurrences of $z^{ \pm 1}$ in the word $w$. Then $\operatorname{Vol}(N) \leq \pi(d(w)-2)$.

Proof This result generalizes [18]. Consider the word $w=q_{1} z^{\epsilon_{1}} q_{2} z^{\epsilon_{2}} \cdots q_{k} z^{\epsilon_{k}}$, where we assume that $\epsilon_{i} \neq 0$, and $q_{i} \in\langle x\rangle \times\langle y\rangle-\{1\}$. Then $d(w)=\sum_{i=1}^{j}\left|\epsilon_{i}\right|$. Let $w_{0}=1$, and for $k=1, \ldots, d(w)$, let $w_{k}(x, y, z)$ be a prefix of $w$ so that $d\left(w_{k}\right)=k$ and $w_{k}$ ends in $z^{ \pm 1}$ (thus $w_{d(w)}=w$ ). Thus $w_{k-1}$ is a prefix of $w_{k}$ such that $w_{k-1}^{-1} w_{k}=q z^{ \pm 1}$ for some $q \in\langle x\rangle \times\langle y\rangle$. Then we may find a string of geodesics $g_{k}$, $k=1, \ldots, d(w)$ in $\mathbb{H}^{3}$ so that $g_{k}$ connects $w_{k-1}(\alpha, \beta, \gamma)(\infty)$ and $w_{k}(\alpha, \beta, \gamma)(\infty)$, and so that $g_{d(w)}$ connects $w_{d(w)-1}(\alpha, \beta, \gamma)(\infty)$ to $\infty=w(\infty)$. We may find a map of a disk $r: D \rightarrow \mathbb{H}^{3}$ with $d(w)$ punctures in $\partial D$ so that $r(\partial D) \subset g_{1} \cup \cdots \cup g_{d}(w)$ by coning each $g_{i}$ to $\infty$, for $1<i<d(w)$. The disk $r(D)$ will be made of $d(w)-2$ triangles, and therefore $\operatorname{Area}(r(D))=\pi(d(w)-2)$. Let $\tilde{g} \subset \mathbb{H}^{3}$ be the geodesic connecting $\infty$ to $\gamma(\infty)$ passing through the self-tangency point of $\tilde{\mathcal{H}}$ with $\gamma(\tilde{\mathcal{H}})$. Let $w_{k-1}^{-1} w_{k}=q z^{ \pm 1}$, for some $q \in\langle x\rangle \times\langle y\rangle$. Then $q^{-1} w_{k-1}(\alpha, \beta, \gamma)^{-1}\left(g_{k}\right)$ connects $\infty$ to $q^{-1} w_{k-1}^{-1} w_{k}(\alpha, \beta, \gamma)(\infty)=\gamma^{ \pm 1}(\infty)$, and we see that $g_{k}$ is a translate of $\tilde{g}$. 
Thus under the projection map $p: \mathbb{H}^{3} \rightarrow N$, each $g_{i}$ will project to the same geodesic $g \subset N$, and the boundary projects to $p(r(\partial D)) \subset g$.

Let $W \subset N$ be a submanifold which is a regular neighborhood of $p(r(D))$ union a small horocusp. Let $U=N \backslash W$, and let $U=\cup_{\alpha} U_{\alpha}$, where each $U_{\alpha}$ is a connected component of $U$. Define a submanifold $U^{\prime} \subset U$ by the property $U_{\alpha} \subset U^{\prime}$ if and only if $\operatorname{im}\left\{\pi_{1}\left(U_{\alpha}\right) \rightarrow \pi_{1}(N)\right\}$ is elementary (that is, the image of the fundamental group is either trivial, $\mathbb{Z}$ or $\mathbb{Z}+\mathbb{Z}$ ). Now, let $N^{\prime} \subset N$ be defined by $N^{\prime}=W \cup U^{\prime}$. Then $\partial N^{\prime} \subset \partial W$, and in particular $\partial N^{\prime}$ is compact. What we will now endeavor to show is that $N=N^{\prime}$. We will let $U^{\prime \prime}=U \backslash U^{\prime}$, so that $N=N^{\prime} \cup U^{\prime \prime}$.

To see how the proof will be finished, suppose that $N^{\prime}$ is irreducible and atoroidal. Then $\partial N^{\prime}$ has no sphere components, and $\chi\left(\partial N^{\prime}\right)<0$, otherwise $\pi_{1}\left(U^{\prime \prime}\right)$ is elementary. Thus, $\pi_{1}\left(N^{\prime}\right)$ is a Kleinian group with $\chi\left(N^{\prime}\right)<0$. Consider the covering space $N^{\prime \prime} \rightarrow N^{\prime}$ such that

$$
\operatorname{im}\left\{\pi_{1}\left(N^{\prime \prime}\right) \rightarrow \pi_{1}\left(N^{\prime}\right)\right\}=\operatorname{im}\left\{\mathbb{Z}^{2} * \mathbb{Z} \rightarrow \pi_{1}\left(N^{\prime}\right)\right\} .
$$

Then $\pi_{1}\left(N^{\prime \prime}\right)$ must also be a bicuspid group. Since $p(r(D))$ gives a relation in $N^{\prime}$ which lifts to $N^{\prime \prime}$, we see that $\pi_{1}\left(N^{\prime \prime}\right)$ is not free. By Lemma 3.1, $N^{\prime \prime}$ must be finitevolume. Since $N^{\prime}$ is atoroidal, each torus component of $\partial N^{\prime \prime}$ must map to a torus boundary component of $N^{\prime}$, which must therefore be a torus boundary component of $N$. This implies that $N^{\prime \prime} \rightarrow N$ is a finite-sheeted cover, which implies that $N^{\prime \prime}=N^{\prime}=N$. Thus, our goal is to show that $N^{\prime}$ is irreducible and atoroidal. This actually might not hold initially, but we modify $N^{\prime}$ until it does hold.

We now prove some properties of the submanifold $N^{\prime}$. Suppose that $S \subset N^{\prime}$ is a 2-sphere. Since $N$ is irreducible, $S=\partial B$, for some ball $B \subset N$. If $B \nsubseteq N^{\prime}$, then $B \cap\left(N-N^{\prime}\right) \neq \varnothing$, so there is a component $U_{\beta} \subset U^{\prime \prime} \subset U$ such that $U_{\beta} \subset B$. But then $\operatorname{im}\left\{\pi_{1}\left(U_{\beta}\right) \rightarrow \pi_{1}(N)\right\}=1$, which contradicts the fact that $U_{\beta} \not \subseteq U^{\prime} \subset N^{\prime}$. Thus, we have shown that $N^{\prime}$ is irreducible.

Now, suppose that $T \subset N^{\prime}$ is 2-torus. Since $N$ is atoroidal, $T$ is separating and either $T$ is parallel to a cusp of $N$, or $T$ is compressible in $N$. In the first case, $T=\partial H$, where $H \cong T \times[0, \infty)$ is a neighborhood of a cusp in $N$. If $H \nsubseteq N^{\prime}$, then there is a component $U_{\beta} \subset H$, in which case $\operatorname{im}\left\{\pi_{1}\left(U_{\beta}\right) \rightarrow \pi_{1}(N)\right\} \leq \operatorname{im}\left\{\pi_{1}(H) \rightarrow \pi_{1}(N)\right\}=$ $\mathbb{Z}+\mathbb{Z}$, which contradicts $U_{\beta} \nsubseteq U^{\prime}$.

Thus, we may assume that $T$ is compressible in $N$. Let $D \subset N \backslash T$ be a compressing disk for $T$, and let $S \subset \mathcal{N}(D \cup T)$ be the 2-sphere component resulting from compressing $T$ by $D$. Then $S=\partial B$, where $B \subset N$ is a ball. There are two possible cases here: either $T \nsubseteq B$ or $T \subset B$. If $T \nsubseteq B$, then $T=\partial Q$, where $Q \subset N$ is a solid torus. If $Q \nsubseteq N^{\prime}$, then there is a component $U_{\beta} \subset Q$. Then 
$\operatorname{im}\left\{\pi_{1}\left(U_{\beta}\right) \rightarrow \pi_{1}(N)\right\} \leq \operatorname{im}\left\{\pi_{1}(Q) \rightarrow \pi_{1}(N)\right\}=\mathbb{Z}$, which contradicts the fact that $U_{\beta} \nsubseteq U^{\prime}$. Thus, we conclude that $Q \subset N^{\prime}$.

In the second case, where $T \subset B$, then $T$ bounds a knot complement $Q$ inside of $B$, since the other side of $T$ is a punctured solid torus in $B$. As before, if $Q \nsubseteq N^{\prime}$, then we conclude that there is a component $U_{\beta} \subset Q \subset B$, which gives a contradiction as before.

Thus, we conclude that every torus $T \subset N^{\prime}$ must either be essential and bound a product neighborhood of a cusp $T=\partial H, H \subset N^{\prime}$, or $T=\partial Q, Q \subset N^{\prime}$, where $Q$ is a (possibly trivial) knot complement. Suppose $Q \subset N^{\prime}$ is a nontrivial knot complement inside of $N^{\prime}$ whose boundary is incompressible in $N^{\prime}$. On $T=\partial Q$, there is a longitude $c \subset T$. In this case, there is a ball $Q \subset B$. Then let $B^{\prime}=B \backslash Q \cup_{T \equiv \partial R} R$, where $R=D^{2} \times S^{1}$ is a solid torus ( $R$ stands for ring) such that the meridian of $R$ is attached to the longitude $c \subset T$ under the identification $T \equiv \partial R$ (really, we should think of $R$ as a trivial knot complement, in which case the meridian is the longitude, and we remove a nontrivial knot complement, and replace it with a trivial one). There is a map $f: B \rightarrow B$ such that $f_{\mid \partial B}$ is the identity, and $f(Q)=R$ (Figure 3). Moreover, this map is homotopy equivalent to the identity map relative to the boundary $\partial B$. We may extend this homotopy equivalence to a map $f: N \rightarrow N$ which is the identity outside of $B$ which is homotopic to the identity.
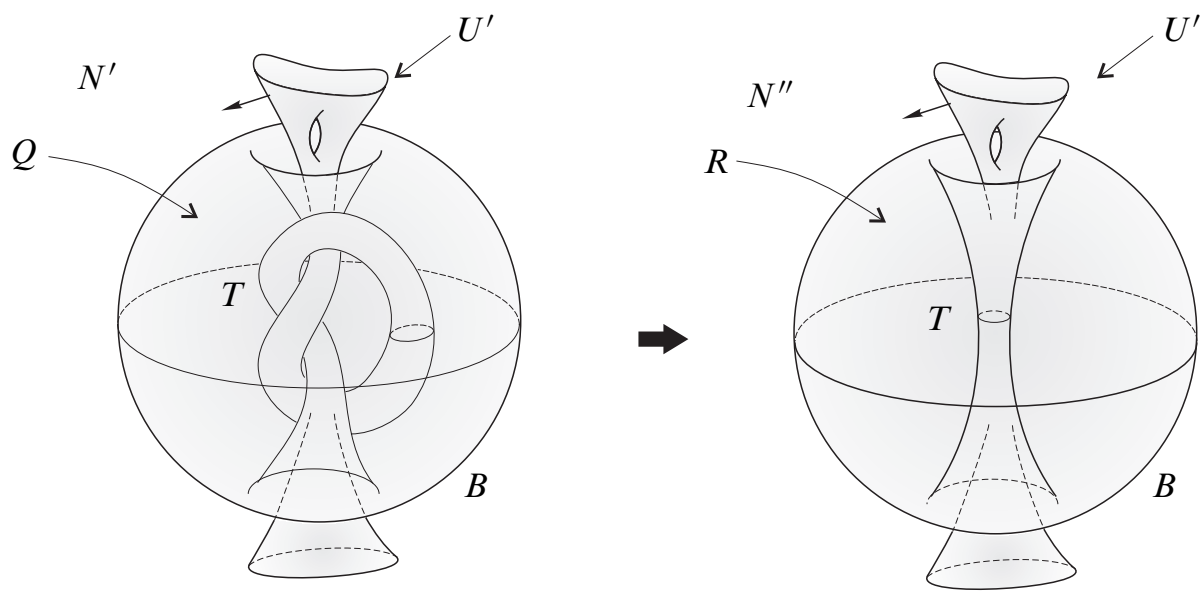

Figure 3: Reimbedding a handle

Let $N^{\prime \prime}=N^{\prime} \backslash Q \cup R$. Then $N^{\prime \prime}=f\left(N^{\prime}\right)$. One may see that $N^{\prime \prime} \subset N$, such that $N \backslash N^{\prime \prime} \cong N \backslash N^{\prime} \cong U^{\prime \prime}$, and that $N^{\prime} \backslash Q \subset N^{\prime}$ is homotopic to $N^{\prime \prime} \backslash R \cong N^{\prime} \backslash Q \subset N$ so that $N \backslash N^{\prime} \equiv N \backslash N^{\prime \prime}$ by a homotopy equivalence. This has the effect of reimbed$\operatorname{ding} U^{\prime \prime}$ in $N$ by a homotopy. Thus, each component $U_{\beta} \subset U^{\prime \prime}$ still has nonelementary 
image of its fundamental group. Moreover, there is a degree one map $f: Q \rightarrow R$, such that $\partial f: \partial Q \rightarrow \partial R$ is a homeomorphism. This map extends to a degree one map $f: N^{\prime} \rightarrow N^{\prime \prime}$. The operation of turning $N^{\prime}$ into $N^{\prime \prime}$ is a version of Fox reimbedding (rather, we reimbed $N^{\prime} \backslash Q \subset N$ by a homotopy equivalence). We now relabel $N_{1}=N^{\prime}, N_{2}=N^{\prime \prime}$, and perform a sequence of reimbeddings, which have associated degree one maps $N_{1} \rightarrow N_{2} \rightarrow N_{3} \rightarrow N_{4} \rightarrow \cdots$ which are homeomorphisms $\partial N_{i} \rightarrow \partial N_{i+1}$. Each $N_{i+1}$ is obtained by replacing a knot complement $Q_{i} \subset N_{i}$ by a solid torus, and therefore $\pi_{1}\left(N_{i}\right) \rightarrow \pi_{1}\left(N_{i+1}\right)$ is not $\pi_{1}$-injective. Since the complementary regions of $N_{i}$ are homotopic for each $i$, we conclude that $N_{i}$ is irreducible. By a result of Soma [43], there exists $i$ such that $N_{i} \rightarrow N_{i+1}$ is a homotopy equivalence, which gives a contradiction. Thus, there exists $N_{n}$ such that every torus $T \subset N_{n}$ which is compressible in $N$ must be compressible in $N_{n}$. Thus, $N_{n}$ is irreducible and atoroidal. Moreover, we have a map $\phi_{n}: N^{\prime} \rightarrow N_{n}$ which induces an epimorphism $\pi_{1}\left(N^{\prime}\right) \rightarrow \pi_{1}(N)$. The argument above shows that $N \backslash N_{n}$ is trivial, which implies that $N=N_{n}=N^{\prime}$, as desired.

Theorem 5.4 Given a rational number $V$, there is an algorithm which will find a finite collection $M_{1}, \ldots, M_{m}$ of finite volume orientable hyperbolic 3-manifolds so that any hyperbolic 3-manifold of volume $<V$ is obtained by Dehn filling on one of the manifolds $M_{i}$.

Proof Let $\varepsilon$ be Margulis' constant for hyperbolic 3-manifolds. By the method of proof of the Jørgensen-Thurston theorem, there is a constant $C$ so that if $M$ is a hyperbolic 3manifold with $\operatorname{Vol}(M)<V$, then $M_{\text {thick( }(\varepsilon)}$ admits a triangulation with $<C V$ tetrahedra (by construction, $C$ is computable from the Margulis constant [33]). The first step of the algorithm is to take $<C V$ tetrahedra, and glue them together in all possible ways to get an orientable manifold with Euler characteristic zero. Next, we run the algorithm described in $[24 ; 48 ; 31]$ to decide which of these manifolds has a hyperbolic interior. Then all hyperbolic 3-manifolds of volume $<V$ will be obtained by Dehn filling on one of the resulting finite collection of manifolds.

The proof of the next theorem actually leads to an independent proof of Theorem 3.4.

Theorem 5.5 There is an algorithm which will determine the finitely many one cusped orientable hyperbolic 3-manifolds $N_{1}, \ldots, N_{k}$ such that $N_{i}$ has two exceptional Dehn fillings of distance $>5$.

Proof We know that if $N$ is a one cusped orientable hyperbolic 3-manifold which has an embedded maximal horocusp neighborhood $\mathcal{H} \subset N$ such that $\operatorname{Vol}(\mathcal{H})>3$, then any 
two Dehn fillings on $N$ have distance $\leq 5$. Thus, we must determine the one cusped orientable hyperbolic 3-manifolds $N$ which have a maximal cusp of volume $\leq 3$ (see the beginning of the proof of Theorem 4.1 for similar logic). By an argument similar to Lemma 3.2 and Theorem 3.4 such manifolds have a non-free bicuspid subgroup $Q=\langle\alpha, \beta, \gamma\rangle$ of finite index in $\pi_{1} N=\Gamma$, such that $\mathcal{H}$ lifts to a maximal cusp of $\mathbb{H}^{3} / Q$. Thus, we must determine the bicuspid groups $Q$ which have a maximal cusp of $\mathbb{H}^{3} / Q$ of volume $\leq 3$. By Theorem 3.4, we know that $\operatorname{Vol}\left(\mathbb{H}^{3} / Q\right)<V(\epsilon)$, for $\epsilon<\pi-3$. But we don't know how to compute $V(\epsilon)$ explicitly. For any $\epsilon<\pi-3$ we show that there is an algorithm which will determine all bicuspid groups $Q$ such that $\mathbb{H}^{3} / Q$ has a maximal cusp of volume $<\pi-\epsilon$.

As in Section 2, we normalize a general bicuspid group $Q=\langle\alpha, \beta, \gamma\rangle$ so that

$$
\alpha=\left(\begin{array}{ll}
1 & a \\
0 & 1
\end{array}\right), \quad \beta=\left(\begin{array}{ll}
1 & b \\
0 & 1
\end{array}\right), \quad \gamma=\left(\begin{array}{cc}
c & -1 \\
1 & 0
\end{array}\right),
$$

where $1 \leq|a| \leq|b| \leq 2 A / \sqrt{3}$, and $|c| \leq|b|$, where $A=\operatorname{Area}(\partial \mathcal{H}) \leq 2(\pi-\epsilon)$. This gives a finite parameter space $\mathcal{P}_{A}$ to search through, and we know that when $A<2 \pi$, the group $Q$ must either be non-free bicuspid by Lemma 3.2, or else $Q$ is indiscrete or not normalized. Furthermore, if $Q$ is discrete but not a free product, then either $Q$ has finite covolume, or $Q$ has torsion. In this case, there will be a nontrivial word $w(x, y, z) \in(\langle x\rangle \times\langle y\rangle) *\langle z\rangle \cong(\mathbb{Z}+\mathbb{Z}) * \mathbb{Z}$ such that $w(\alpha, \beta, \gamma)=1 \in Q$. A lift of $w(\alpha, \beta, \gamma)$ to $\mathrm{SL}_{2} \mathbb{C}$ gives a matrix

such that

$$
\begin{aligned}
& W(a, b, c)=\left(\begin{array}{cc}
* & * \\
p(a, b, c) & *
\end{array}\right), \\
& W(a, b, c)=\left(\begin{array}{ll}
1 & 0 \\
0 & 1
\end{array}\right)
\end{aligned}
$$

when the variables $(a, b, c)$ correspond to the group $Q$. The lower left entry of $W(a, b, c)$ may be regarded as a polynomial $p(a, b, c) \in \mathbb{Z}[a, b, c]$ which vanishes on the parameters corresponding to $Q$.

If

$$
\mu=\left(\begin{array}{ll}
w & x \\
y & z
\end{array}\right)
$$

represents a matrix in $\mathrm{SL}_{2} \mathbb{C}$, and if $\tilde{\mathcal{H}}$ is the horoball of height 1 centered at $\infty$ in $\mathbb{H}^{3}$, then $\mu(\tilde{\mathcal{H}})$ is a horoball of height $1 /|y|^{2}$ for $y \neq 0$. Thus, if $\tilde{\mathcal{H}} \cap \mu(\tilde{\mathcal{H}})=\varnothing$, we see that $|y| \geq 1$. So, if $\mu \in Q$ is an element of the bicuspid group $Q$, and $\tilde{\mathcal{H}}$ is precisely invariant under $Q$, then we see that if $|y|<1$, we must have $\tilde{\mathcal{H}}=\mu(\tilde{\mathcal{H}})$, and therefore $\mu$ is a parabolic element, so $y=0$. 
Similarly, if $Q$ is indiscrete, then the closure $\bar{Q} \leq \mathrm{SL}_{2} \mathbb{C}$ must be dense in a Lie subgroup of $\mathrm{SL}_{2} \mathbb{C}$, and thus there are elements $w(\alpha, \beta, \gamma) \in Q$ such that $|W(a, b, c)-I|^{2}<\epsilon$, for any small $\epsilon$. In particular, if

$$
W(a, b, c)=\left(\begin{array}{cc}
* & * \\
p(a, b, c) & *
\end{array}\right)
$$

where $p(a, b, c)<1$, then either $p(a, b, c)=0$, or the three generator group associated to the parameters $(a, b, c)$ does not have an embedded horocusp $\mathcal{H} \subset \mathbb{H}^{3} /\langle\alpha, \beta, \gamma\rangle$ which is a projection of a horoball at height 1 in $\mathbb{H}^{3}$, and is therefore not bicuspid with normalized generators. Find a finite collection of such polynomials $\left\{p_{1}, \ldots, p_{k}\right\} \in$ $\mathbb{Z}[a, b, c]$ so that the sets $\left\{(a, b, c) \mid p_{i}(a, b, c)<1\right\}$ cover $\mathcal{P}_{A}$. Each such polynomial is determined by a word $w_{i}(x, y, z)$. We may find a finite collection since $\mathcal{P}_{A}$ has compact closure. To make this search algorithmic, enumerate $p(a, b, c)$ for all $w(x, y, z) \in$ $\mathbb{Z}^{2} * \mathbb{Z}$. Cover $\mathcal{P}_{A}$ by compact subsets, such as cubes with dyadic vertices and sidelength $2^{-n}$, such that each cube $C \subset \mathcal{P}_{A+\delta}$ (where $A+\delta<2 \pi$ ), so that we will cover all bicuspid groups with $\operatorname{Vol}(\mathcal{H}) \leq 3$ and still exclude free bicuspid groups. For a given polynomial $p(a, b, c)$, and a given cube $C$, one may determine whether $|p(a, b, c)|<1$ for all points $(a, b, c) \in C$, by determining the maximal value of $p(a, b, c)$ on $C$, which is algorithmic (an interior maximum is determined by computing a point where the gradient $\nabla p(a, b, c)=0$, which may be computed via algebraic geometry, whereas boundary maxima may be determined by Lagrange multipliers inductively on the faces of the cube). Inductively, we refine the covering and increase the number of polynomials, until we find a covering of $\mathcal{P}_{A}$ (this is similar to the process employed in [21, Proposition 1.28]).

All of the discrete non-free bicuspid groups with a cusp of volume $\leq 3$ will occur somewhere in the parameter space $\mathcal{P}_{A}$ with some $w_{i}(\alpha, \beta, \gamma)$ parabolic, and therefore $p_{i}(a, b, c)=0$. Conversely, if $(a, b, c) \in \mathcal{P}_{A}$ is a point where $p_{i}(a, b, c)=0$, then it either corresponds to an irreducible bicuspid representation of $\mathbb{Z}^{2} * \mathbb{Z}$ when $W(a, b, c)$ is parabolic or trivial, or gives an indiscrete representation, or a representation with torsion, or it may correspond to a bicuspid group whose generators have not been normalized. If $0<p_{i}(a, b, c)<1$, then either the group is indiscrete, or it does not have a maximal cusp $\mathcal{H}$ normalized as above, so does not correspond to a bicuspid group. If $p_{i}(a, b, c)=0$ and the associated bicuspid group is discrete, then $w_{i}(a, b, c)=\alpha^{m} \beta^{n}$. We may apply Lemma 5.3 to the word $w^{\prime}=w_{i} \alpha^{-m} \beta^{-n}$ to conclude that the covolume of the discrete torsion-free $Q$ will be bounded by the complexity $\pi\left(d\left(w_{i}\right)-2\right)=$ $\pi\left(d\left(w^{\prime}\right)-2\right)$. Let $V$ be the supremum of these numbers. Thus, we have shown the existence of an algorithm to determine a bound on the covolume of bicuspid groups with a maximal cusp of volume $\leq \pi-\epsilon$. Moreover, this gives another proof of Theorem 3.4. 
The next step is to enumerate all orientable hyperbolic manifolds with volume $<V$. We use Theorem 5.4 to give a finite collection $M_{1}, \ldots, M_{m}$ of finite volume hyperbolic 3manifolds so that any hyperbolic 3-manifold of volume $<V$ is obtained by hyperbolic Dehn filling on one of the manifolds $M_{j}$. Finally, we use Theorem 5.1 to determine the set of all exceptional Dehn fillings on each $M_{j}$. We then search this list of exceptional Dehn fillings for any one cusped manifolds with two exceptional fillings of distance $>5$. By Theorem 4.1, there will be only finitely many isometry types of hyperbolic one cusped manifolds with two exceptional Dehn fillings of distance $>5$.

\section{Conclusion}

There are several problems suggested by the results in this paper. Of course, there is Gordon's conjectured classification of one cusped manifolds with two exceptional fillings of distance $>5$ (see [27, Problem 1.77, Conjecture (B)]). As we point out, it will suffice to classify the manifolds with a cusp of volume $\leq 3$. Searching through the SnapPea census of cusped orientable manifolds [47], there appears to be few one or two cusped manifolds which have cusp volume $\leq 3$ and which are not obtained by Dehn filling on a manifold with corresponding cusp of volume very close to 3 and which have fewer than three cusps. The collection of three cusped manifolds with a cusp of volume $\leq 3$ is finite, as follows from the argument of Theorem 4.1. We show several examples of three cusped manifolds with a cusp of volume $\leq 3$ in Figure 4, and which
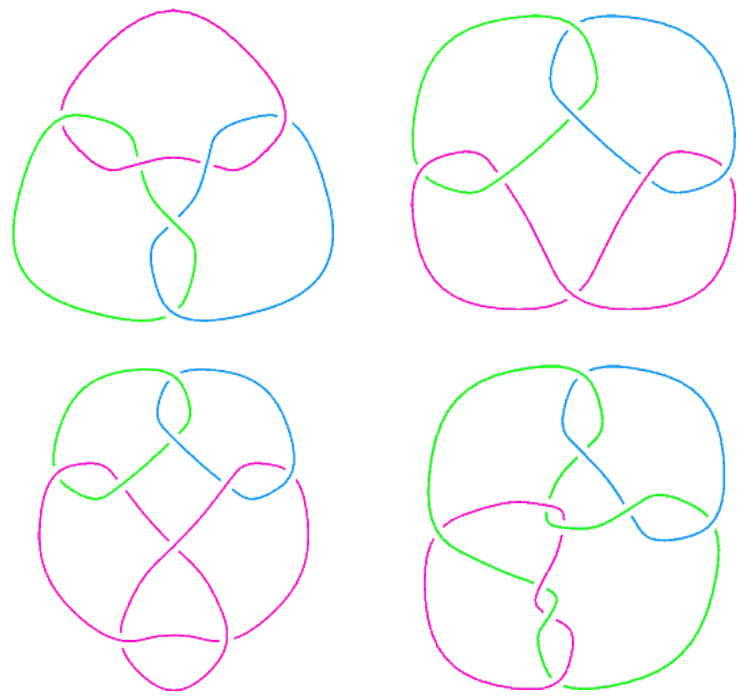

Figure 4: Some 3 component links with cusps of volume $\leq 3$ 
give rise to infinite families of 3 -manifolds with maximal cusps of volume $\leq 3$ via Dehn filling. We remark that if one wants to classify manifolds with $>8$ exceptional Dehn fillings, it suffices to classify manifolds with two exceptional Dehn fillings $r_{1}, r_{2}$ with $\Delta\left(r_{1}, r_{2}\right)>6$. Thus, it suffices to consider manifolds with a cusp of volume $\leq 2 \frac{4}{7}$ corresponding to the parameter space $\mathcal{P}_{5 \frac{1}{7}}$. This would significantly simplify the search space in the algorithm proposed in Theorem 5.5.

\section{References}

[1] C C Adams, The noncompact hyperbolic 3-manifold of minimal volume, Proc. Amer. Math. Soc. 100 (1987) 601-606 MR894423

[2] I Agol, Tameness of hyperbolic 3-manifolds arXiv:math/0405568

[3] I Agol, Bounds on exceptional Dehn filling, Geom. Topol. 4 (2000) 431-449 MR1799796

[4] I Agol, M Culler, P B Shalen, Dehn surgery, homology and hyperbolic volume, Algebr. Geom. Topol. 6 (2006) 2297-2312 MR2286027

[5] J W Anderson, RD Canary, M Culler, PB Shalen, Free Kleinian groups and volumes of hyperbolic 3-manifolds, J. Differential Geom. 43 (1996) 738-782 MR1412683

[6] L Bers, Spaces of Kleinian groups, from: "Several Complex Variables, I (Proc. Conf., Univ. of Maryland, College Park, Md., 1970)”, Springer, Berlin (1970) 9-34 MR0271333

[7] S A Bleiler, C D Hodgson, Spherical space forms and Dehn filling, Topology 35 (1996) 809-833 MR1396779

[8] F Bonahon, J-P Otal, Variétés hyperboliques à géodésiques arbitrairement courtes, Bull. London Math. Soc. 20 (1988) 255-261 MR931188

[9] B H Bowditch, End invariants of hyperbolic 3-manifolds, Preprint (2005) Available at http://www.warwick.ac.uk/ masgak/preprints.html

[10] B H Bowditch, Notes on tameness, Preprint (2006) Available at http:// www. warwick.ac.uk/ masgak/preprints.html

[11] J F Brock, K W Bromberg, On the density of geometrically finite Kleinian groups, Acta Math. 192 (2004) 33-93 MR2079598

[12] J F Brock, R D Canary, Y N Minsky, Classification of Kleinian surface groups II: the ending lamination conjecture arXiv:math/0412006 1

[13] K Bromberg, Projective structures with degenerate holonomy and the Bers density conjecture, Ann. of Math. (2) 166 (2007) 77-93 MR2342691

[14] D Calegari, D Gabai, Shrinkwrapping and the taming of hyperbolic 3-manifolds, J. Amer. Math. Soc. 19 (2006) 385-446 MR2188131

[15] C Cao, G R Meyerhoff, The orientable cusped hyperbolic 3-manifolds of minimum volume, Invent. Math. 146 (2001) 451-478 MR1869847 
[16] H-D Cao, X-P Zhu, A complete proof of the Poincaré and geometrization conjecturesapplication of the Hamilton-Perelman theory of the Ricci flow, Asian J. Math. 10 (2006) 165-492 MR2233789

[17] T D Comar, Hyperbolic Dehn surgery and convergence of Kleinian groups, ProQuest LLC, Ann Arbor, MI (1996) PhD Thesis-University of Michigan

[18] D Cooper, The volume of a closed hyperbolic 3-manifold is bounded by $\pi$ times the length of any presentation of its fundamental group, Proc. Amer. Math. Soc. 127 (1999) 941-942 MR1646313

[19] M Culler, PB Shalen, Paradoxical decompositions, 2-generator Kleinian groups, and volumes of hyperbolic 3-manifolds, J. Amer. Math. Soc. 5 (1992) 231-288 MR1135928

[20] D Gabai, R Meyerhoff, P Milley, Minimum volume cusped hyperbolic three-manifolds, J. Amer. Math. Soc. 22 (2009) 1157-1215 MR2525782

[21] D Gabai, R Meyerhoff, N Thurston, Homotopy hyperbolic 3-manifolds are hyperbolic, Ann. of Math. (2) 157 (2003) 335-431 MR1973051

[22] CM Gordon, Boundary slopes of punctured tori in 3-manifolds, Trans. Amer. Math. Soc. 350 (1998) 1713-1790 MR1390037

[23] C M Gordon, Dehn filling: a survey, from: "Knot theory (Warsaw, 1995)", (V F R Jones, J H Kania-Bartoszyńska, J Przytycki, P Traczyk, V G Turaev, editors), Banach Center Publ. 42, Polish Acad. Sci., Warsaw (1998) 129-144 MR1634453

[24] M Hildebrand, J Weeks, A computer generated census of cusped hyperbolic 3manifolds, from: "Computers and mathematics (Cambridge, MA, 1989)", (E Kaltofen, S M Watt, editors), Springer, New York (1989) 53-59 MR1005959

[25] W H Jaco, P B Shalen, Seifert fibered spaces in 3-manifolds, Mem. Amer. Math. Soc. 21 (1979) viii+192 MR539411

[26] T Jørgensen, On pairs of once-punctured tori, from: "Kleinian groups and hyperbolic 3-manifolds (Warwick, 2001)", (Y Komori, V Markovic, C Series, editors), London Math. Soc. Lecture Note Ser. 299, Cambridge Univ. Press (2003) 183-207 MR2044551

[27] R Kirby, editor, Problems in low-dimensional topology, from: "Geometric topology (Athens, GA, 1993)", (W H Kazez, editor), AMS/IP Stud. Adv. Math. 2, Amer. Math. Soc. (1997) 35-473 MR1470751

[28] B Kleiner, J Lott, Notes on Perelman's papers, Geom. Topol. 12 (2008) 2587-2855 MR2460872

[29] M Lackenby, Word hyperbolic Dehn surgery, Invent. Math. 140 (2000) 243-282 MR1756996

[30] M Lackenby, R Meyerhoff, The maximal number of exceptional Dehn surgeries arXiv:0808.1176

[31] J Manning, Algorithmic detection and description of hyperbolic structures on closed 3-manifolds with solvable word problem, Geom. Topol. 6 (2002) 1-25 MR1885587

[32] A Marden, The geometry of finitely generated kleinian groups, Ann. of Math. (2) 99 (1974) 383-462 MR0349992 
[33] R Meyerhoff, A lower bound for the volume of hyperbolic 3-manifolds, Canad. J. Math. 39 (1987) 1038-1056 MR918586

[34] Y Minsky, The classification of Kleinian surface groups. I. Models and bounds, Ann. of Math. (2) 171 (2010) 1-107 MR2630036

[35] J Morgan, G Tian, Ricci flow and the Poincaré conjecture, Clay Math. Monogr. 3, Amer. Math. Soc. (2007) MR2334563

[36] H Namazi, J Souto, Non-realizability and ending laminations: Proof of the Density Conjecture, Preprint (2010) Available at http://www.ma.utexas.edu/users/ hossein/contents/ending6.6.pdf

[37] K Ohshika, Realising end invariants by limits of minimally parabolic, geometrically finite groups arXiv:math/0504546

[38] J-P Otal, Le théorème d'hyperbolisation pour les variétés fibrées de dimension 3, Astérisque 235, Soc. Math. France (1996) MR1402300

[39] G Perelman, The entropy formula for the Ricci flow and its geometric applications arXiv:math.DG/0211159

[40] G Perelman, Ricci flow with surgery on three-manifolds arXiv:math.DG/0303109

[41] S Rafalski, Immersed turnovers in hyperbolic 3-orbifolds, Groups Geom. Dyn. 4 (2010) 333-376 MR2595095

[42] R Riley, Applications of a computer implementation of Poincaré's theorem on fundamental polyhedra, Math. Comp. 40 (1983) 607-632 MR689477

[43] T Soma, Sequences of degree-one maps between geometric 3-manifolds, Math. Ann. 316 (2000) 733-742 MR1758451

[44] T Soma, Existence of ruled wrappings in hyperbolic 3-manifolds, Geom. Topol. 10 (2006) 1173-1184 MR2255495

[45] W P Thurston, Hyperbolic structures on 3-manifolds, II: Surface groups and 3manifolds which fiber over the circle arXiv:math/9801045

[46] W P Thurston, The geometry and topology of three-manifolds, Princeton Univ. Math. Dept. Lecture Notes (1979) Available at http://msri.org/publications/books/ gt $3 \mathrm{~m} /$

[47] J R Weeks, SnapPea: A computer program for creating and studying hyperbolic 3manifolds Available at http://www.geometrygames.org/SnapPea/

[48] J R Weeks, Convex hulls and isometries of cusped hyperbolic 3-manifolds, Topology Appl. 52 (1993) 127-149 MR1241189

Department of Mathematics, University of California, Berkeley, CA 94720-3840, USA ianagol@math. berkeley . edu

Proposed: Cameron Gordon

Seconded: Joan Birman, David Gabai

Received: 19 March 2008

Revised: 12 June 2010 